\title{
Painful Bladder Syndrome: An Unusual Presentation in a Case of Upper Tract Fungus Balls
}

\author{
Petar Bajic Jessica Wetterlin Larissa Bresler \\ Department of Urology, Loyola University Medical Center, Maywood, III., USA
}

\section{Key Words}

Funguria $•$ Fungus balls $•$ Painful bladder syndrome . Urinary tract infection

\begin{abstract}
Urinary tract fungus balls are a rare pathologic entity which may be asymptomatic or have variable presentations. To date, there have been no documented cases of fungus balls presenting as painful bladder syndrome. Painful bladder syndrome is a constellation of symptoms which may include pelvic pain, urgency and frequency not explained by other causes. Here, we present the first case of these two entities concurrently. Our patient had a longstanding history of diabetes, nephrolithiasis and recurrent urinary tract infections. He presented with symptoms of painful bladder syndrome and work-up revealed filling defects within the renal collecting system concerning for malignancy. Subsequent ureteroscopy revealed dense white debris consistent with candida fungus balls. Following clearance of the debris and antifungal therapy, our patient has remained asymptomatic.
\end{abstract}

Copyright $\odot 2015$ S. Karger AG, Basel

\section{KARGER}

Fax +41613061234

E-Mail karger@karger.ch

www.karger.com
(C) 2015 S. Karger AG, Basel

1015-9770/14/0092-0104\$38.00/0

Accessible online at:

www.karger.com/cur
JS is an 87 year old diabetic male with complex history including multiple urologic interventions for uric acid nephrolithiasis. He has complained of dysuria, frequency, urgency and suprapubic pain starting 5 years prior to his first stone procedure. His voiding complaints persisted even with negative urine cultures and he was subsequently lost to follow-up, his symptoms having been attributed to painful bladder syndrome (PBS).

He returned to clinic years later with similar complaints. He denied hematuria. Workup included urinalysis which revealed positive leukocyte esterase, negative nitrites, greater than 50 red blood cells and greater than 50 white blood cells. Urine culture revealed no growth.

Office cystoscopy revealed a small, otherwise normal bladder with diffuse erythema. CT urogram revealed a filling defect in a right renal calyx and a small stone. He went to the operating room where ureteroscopy revealed a lower pole calyceal diverticulum with a small stone and debris. Intraoperative cytology was negative for malignancy. Stone analysis showed uric acid and calcium oxalate.

His post-operative course was uncomplicated but he continued to experience vague urinary symptoms over the next year. He had intermittent urinary tract infections treated with antibiotics. Post-void residuals were within normal limits. Urodynamics was recommended but the patient refused.

A renal ultrasound was performed one year later which revealed a $5 \mathrm{~mm}$ right lower pole calculus with mild hydronephrosis. CT urogram was repeated which revealed multiple upper tract filling defects (fig. 1).

Petar Bajic

Department of Urology, Loyola University Medical Center $859 \mathrm{~W}$ Erie st Apt 506

IL-60642, Chicago (USA)

E-Mail pbajic@lumc.edu 


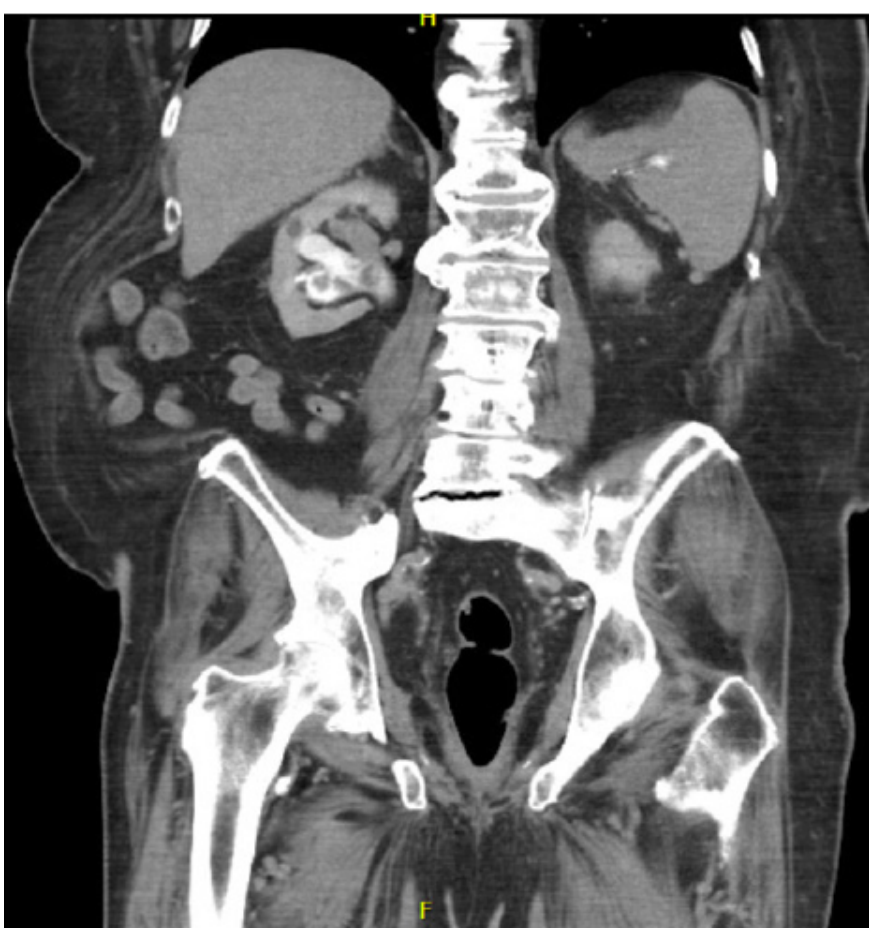

Fig. 1. Delayed phase of contrast CT revealing filling defects in the right upper pole.

The patient returned to the operating room where ureteroscopy revealed dense white debris throughout the collecting system, which was extracted along with 2 stones. A double-J ureteral stent and Foley catheter were placed.

Right upper tract cytology showed numerous neutrophils and fungal forms. Pathologic analysis and culture of the debris revealed candida glabrata fungus balls. The patient was started on intravenous amphotericin B and flucytosine and his symptoms rapidly improved. He did not tolerate intravesical amphotericin due to pain.
After 4 weeks of antifungal therapy, CT scan revealed no residual disease. His ureteral stent and Foley catheter were removed and he remains asymptomatic on prophylactic fluconazole.

Candiduria is common amongst urologic patients with risk factors including indwelling urinary catheters and stents $(83 \%)$, prior antibiotic therapy $(90 \%)$, diabetes $(39 \%)$, urinary tract pathology $(38 \%)$ and malignancy (22\%) [1]. Eleven percent of patients with candiduria do not have predisposing factors. Candiduria is associated with Candida albicans in $52 \%$ of cases, and candida glabrata in $16 \%$ [1]. Candida glabrata is associated with fluconazole resistance in $20-75 \%$ of strains [2].

Upper tract candida infections typically occur in the setting of obstruction and may result in fungus balls [3]. The gold standard treatment for fungus balls is fluconazole or amphotericin B with or without flucytosine. For candida glabrata infections, fluconazole resistance remains prominent but may be overcome by higher doses or alternative antimicrobials [3]. Therapy should continue until the fungus balls are removed, symptoms resolve and fungal cultures are negative. Amphotericin irrigation may be used via Foley catheter or nephrostomy tube as an adjunct or monotherapy [4]

Our patient's unusual presentation is a reminder that symptoms consistent with PBS may be a result of a treatable underlying pathology. The timing of when the patient developed fungus balls is unclear but he his symptoms started 5 years prior to any urologic surgery. Given his response to treatment it appears that these symptoms which had been attributed to PBS were actually due to a fungal infection. When assessing the patient with vague urinary symptoms, differential diagnoses should be broad and the entire genitourinary tract must be considered. Comprehensive evaluation should be performed to rule out rare pathologic entities such as those discovered in this case.

\section{References}

1 Kauffman CA, Vazquez JA, Sobel JD, Gallis HA, McKinsey DS, Karchmer AW, Sugar AM, Sharkey PK, Wise GJ, Mangi R, Mosher A, Lee JY, Dismukes WE: Prospective multicenter surveillance study of funguria in hospitalized patients. The national Institute for Allergy and Infectious Diseases (NIAID) Mycoses Study Group. Clin Infect Dis 2000; 30:14-18.
2 Spellberg BJ, Filler SG, Edwards JE Jr: Current treatment strategies for disseminated candidiasis. Clin Infect Dis 2006;42:244251

3 Irby PB, Stoller ML, McAninch JW: Fungal bezoars of the upper urinary tract. J Urol 1990;143:447-451
4 Bisht V, Voort JV: Clinical practice: Obstructive renal candidiasis in infancy. Eur J Pediatr 2011;170:1227-1235. 